University of Warwick institutional repository: http://go.warwick.ac.uk/wrap This paper is made available online in accordance with publisher policies. Please scroll down to view the document itself. Please refer to the repository record for this item and our policy information available from the repository home page for further information.

To see the final version of this paper please visit the publisher's website. Access to the published version may require a subscription.

Author(s): P. Sullivan, T. S. Jones, A. J. Ferguson and S. Heutz Article Title: Structural templating as a route to improved photovoltaic performance in copper phthalocyanine/fullerene $\left(\mathrm{C}_{60}\right)$ heterojunctions Year of publication: 2007

Link to published version: http://dx.doi.org/ 10.1063/1.2821229

Publisher statement: None 


\title{
Structural templating as a route to improved photovoltaic performance in copper phthalocyanine/fullerene $\left(\mathrm{C}_{60}\right)$ heterojunctions
}

\author{
P. Sullivan and T. S. Jones ${ }^{a)}$ \\ Department of Chemistry, University of Warwick, Coventry, CV4 7AL, United Kingdom
}

\author{
A. J. Ferguson \\ Department of Chemistry, Imperial College London, London SW7 2AZ, United Kingdom \\ S. Heutz \\ London Centre for Nanotechnology, Department of Materials, Imperial College London, London SW7 2AZ, \\ United Kingdom
}

(Received 9 August 2007; accepted 13 November 2007; published online 6 December 2007)

\begin{abstract}
We have developed a method to improve the short circuit current density in copper phthalocyanine $(\mathrm{CuPc}) /$ fullerene $\left(\mathrm{C}_{60}\right)$ organic solar cells by $\sim 60 \%$ by modifying the CuPc crystal orientation through use of a molecular interlayer to maximize charge transport in the direction between the two electrodes. Powder x-ray diffraction and electronic absorption spectroscopy show that a thin 3,4,9,10-perylenetetracarboxylic dianhydride interlayer before $\mathrm{CuPc}$ growth templates the $\mathrm{CuPc}$ film structure, forcing the molecules to lie flat with respect to the substrate surface, although the intrastack orientation is unaffected. This modified stacking configuration facilitates charge transport and improves charge collection. (C) 2007 American Institute of Physics. [DOI: 10.1063/1.2821229]
\end{abstract}

Considerable effort is being directed at the development of small-molecule organic photovoltaic (OPV) devices using materials such as copper phthalocyanine $(\mathrm{CuPc})$ as both the light absorbing and hole-transport layer. ${ }^{1-3}$ Fullerene $\left(\mathrm{C}_{60}\right)$ has become the preferred electron acceptor and it has been shown that cell structures containing mixed donor-acceptor layers, fabricated by codeposition of $\mathrm{CuPc}$ and $\mathrm{C}_{60}$, show significant improvement in device performance over simple bilayer heterojunctions. ${ }^{4-6}$ Although $\mathrm{CuPc} / \mathrm{C}_{60}$ OPV devices have reached power conversion efficiencies $\left(\eta_{p}\right)$ of $\sim 5 \%,{ }^{5}$ their performance remains limited by the low exciton diffusion length of $\mathrm{CuPc}$ and poor charge transport in the mixed $\mathrm{CuPc} / \mathrm{C}_{60}$ layers.

The $\alpha$-polymorph of CuPc, which is formed when films are grown on noninteracting substrates at room temperature, displays strongly anisotropic charge mobility as a result of the formation of one-dimensional (1D) stacks of molecules. ${ }^{7,8}$ The molecules in these stacks align with their molecular planes parallel to each other, tilted by approximately $65^{\circ}$ to the stacking axis, with the stacks oriented such that a "herringbone" arrangement is adopted by molecules in neighboring columns. The peak charge mobility occurs along the stacking axis due to the strong $\pi$-coupling between neighboring molecules, with charge transport between columns limited by a hopping mechanism. The 1D stacks within the polycrystallites are preferentially oriented with the stacking axis parallel to the substrate surface, resulting in a peak charge mobility parallel to the substrate of around 4.13 $\times 10^{-3} \mathrm{~cm}^{2} / \mathrm{V} \mathrm{s}{ }^{9}$ However, in a standard OPV cell, the desired direction of charge transport is perpendicular to the substrate surface and, hence, device performance is limited by the hopping of charges between neighboring stacks. Mobilities around four orders of magnitude lower at 5 $\times 10^{-7} \mathrm{~cm}^{2} / \mathrm{V} \mathrm{s}$ have been reported for this orientation. ${ }^{10} \mathrm{In}$ order to achieve maximum mobility in CuPc donor layers, it

${ }^{a)}$ FAX: +44 (0)24 7652 4112. Electronic mail: t.s.jones@warwick.ac.uk. would be desirable for the $1 \mathrm{D}$ stacks to be rotated so that the stacking direction lies parallel to the surface normal.

It is well-known that 3,4,9,10-perylenetetracarboxylic dianhydride (PTCDA) molecules lie approximately parallel to the substrate surface when deposited onto noninteracting substrates. $^{11,12}$ It has also been reported that the initial growth of a thin layer of PTCDA causes a subsequent freebase phthalocyanine $\left(\mathrm{H}_{2} \mathrm{Pc}\right)$ layer to follow the underlying structure rather than adopting its usual herringbone structure, ${ }^{13-15}$ an effect referred to as structural templating. This templating effect is caused by an interaction between the $\pi$-orbitals of the PTCDA underlayer and the $\mathrm{H}_{2} \mathrm{Pc}$ molecules, and is long range with no sign of the reappearance of the $\mathrm{H}_{2} \mathrm{Pc}$ herringbone structure in films as thick as $380 \mathrm{~nm} .{ }^{13,14}$ In this letter, we show that this templating effect can also be used for CuPc films in an OPV heterojunction cell with significant increase in the short circuit current density $\left(J_{\mathrm{SC}}\right)$. The results demonstrate the effectiveness of tailoring the structure of the CuPc films for OPV applications.

The structures were fabricated on commercially available indium-tin-oxide (ITO) coated glass substrates (CRL Opto) after initial solvent cleaning. The organic layers were grown by vacuum deposition in a Kurt J. Lesker Spectros system with a base pressure of about $8 \times 10^{-8}$ mbar. The organic materials used in the devices CuPc (Aldrich 97\%), $\mathrm{C}_{60}$ (MER Corp. 99.5\%), bathocuproine (Aldrich 98\%), and PTCDA (Aldrich 97\%) were twice purified by thermal gradient sublimation prior to deposition. A deposition rate of $1 \AA \mathrm{s}^{-1}$ was used for all organic layers except PTCDA, which was grown at $0.1-0.2 \AA \mathrm{s}^{-1}$. The $\mathrm{Al}$ cathodes were deposited in situ by evaporation through a shadow mask to a thickness of $1000 \AA$, giving an active area of $0.16 \mathrm{~cm}^{2}$. X-ray diffraction (XRD) traces were obtained from a Philips PW1700 series automated powder diffractometer using $\mathrm{Cu} \mathrm{K} \alpha$ radiation, whilst electronic absorption spectra were measured using a Perkin Elmer Lambda 2 spectrometer. $J-V$ curves were measured with a Keithley 2400 sourcemeter un- 


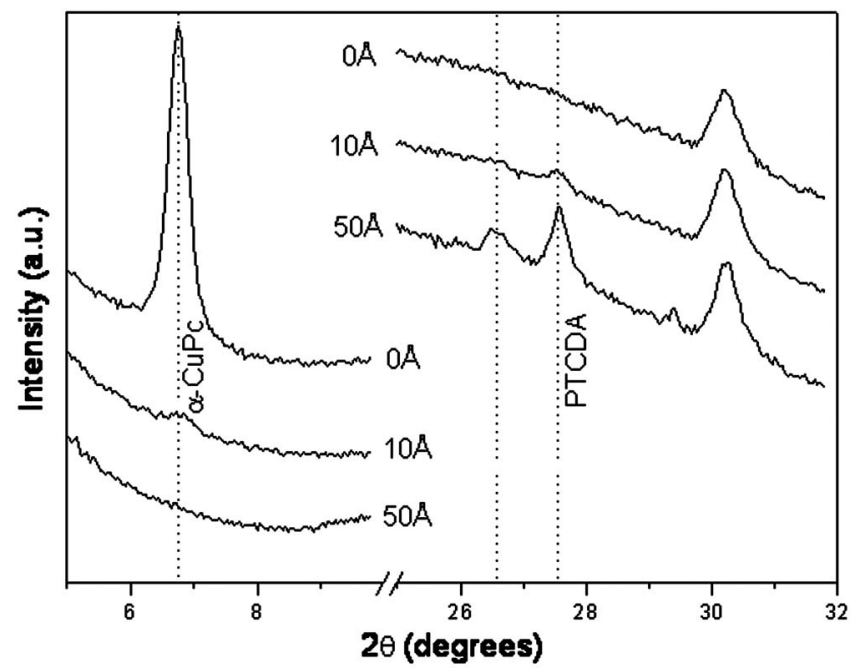

FIG. 1. Powder XRD patterns for a $300 \AA$ CuPc layer grown on PTCDA interlayers of thicknesses between 0 and $50 \AA$. The peak at $\sim 30.2^{\circ}$ is due to the ITO coating and is used as a reference for peak position and height, while the sloped background is due to the glass substrate.

der AM1.5 solar illumination at $100 \mathrm{~mW} \mathrm{~cm}^{-2}$ (1 sun) from a Sciencetech solar simulator.

Powder XRD traces for $300 \AA \mathrm{CuPc}$ layers grown on top of PTCDA interlayers of thicknesses ranging from $0-50 \AA$ on ITO substrates are shown in Fig. 1. With no PTCDA interlayer, the $\mathrm{CuPc}$ exhibits a single intense diffraction feature centered at $6.8^{\circ}$, corresponding to diffraction from the (200) plane of $\alpha$-CuPc, and consistent with the stacking axis lying parallel to the substrate. ${ }^{16,17}$ Inclusion of a PTCDA interlayer results in a peak appearing at $\sim 27.6^{\circ}$, corresponding to diffraction from the (102) plane of PTCDA, but is also accompanied by a substantial change in the rest of the diffraction pattern. On increasing the PTCDA layer thickness, the intensity of the $\alpha$-CuPc peak at $6.8^{\circ}$ is severely diminished, until disappearing completely at $50 \AA$. This suggests that the underlying PTCDA layer has a considerable effect on the structure of the CuPc layer. The disappearance of this peak is accompanied by the gradual emergence of a different peak at $\sim 26.6^{\circ}$, corresponding to an interplanar separation of $3.33 \AA$, which cannot be explained by the presence of the PTCDA layer or by a reorientation of the bulk $\mathrm{CuPc}$ herringbone structure since the peak is not present for $\alpha$-CuPc in powder form. ${ }^{18}$ Instead, it appears that the PTCDA has forced the CuPc layer to adopt a completely different structure.

Electronic absorption spectra of a $300 \AA \mathrm{CuPc}$ layer grown directly on ITO, and 300 and $1000 \AA \mathrm{CuPc}$ layers grown on a $20 \AA$ PTCDA interlayer are shown in Fig. 2. The nontemplated $\mathrm{CuPc}$ layer shows a spectrum typical of $\alpha$-phase $\mathrm{CuPc}$, with the main absorption displaying peaks at 694 and $622 \mathrm{~nm}$ as a result of aggregation. It is known that the relative intensity of the peak at $694 \mathrm{~nm}$ is indicative of the level of monomer character of the film. ${ }^{4,19}$ The only significant change in the absorption features on inclusion of a $20 \AA$ PTCDA interlayer is the relative intensity of the $694 \mathrm{~nm}$ peak, suggesting that the dominant electronic interactions between $\mathrm{CuPc}$ molecules are unaffected by the structural templating effect imparted by the PTCDA underlayer, and that the 1D stacks remain intact in the modified structure. The $694 \mathrm{~nm}$ peak shows a decrease in relative intensity on inclusion of the $20 \AA$ PTCDA interlayer and a further

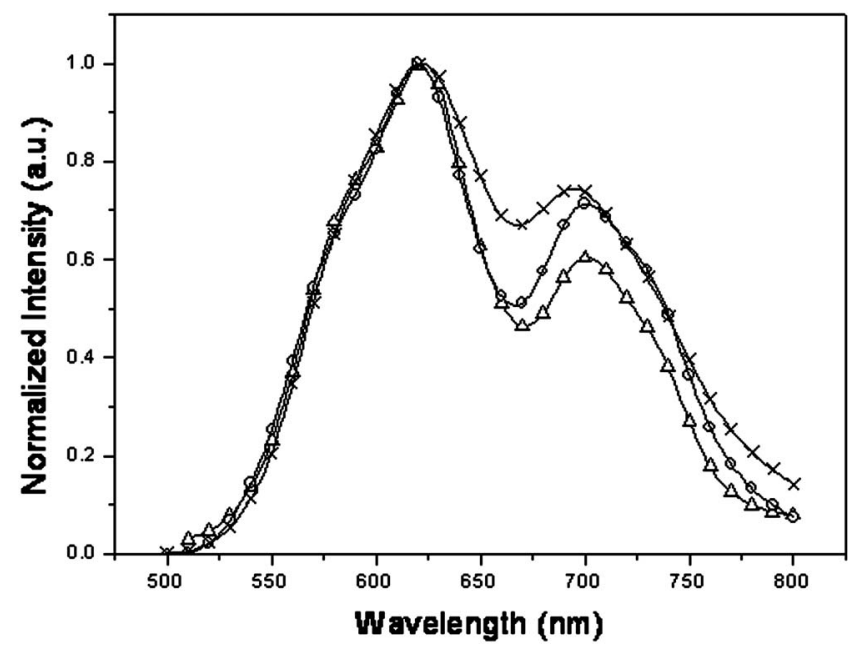

FIG. 2. Electronic absorption spectra of a $300 \AA$ CuPc layer grown directly on ITO (crosses) and on a $20 \AA$ PTCDA interlayer (empty circles), and a $1000 \AA$ CuPc layer grown on a $20 \AA$ PTCDA interlayer (empty triangles).

significant decrease with an increase in the thickness of the templated $\mathrm{CuPc}$ layer. This suggests that a higher proportion of the CuPc molecules are able to form aggregate stacks in the templated layers, and is evidence of increased order imparted in the structure by the underlying PTCDA layer. This effect is increasingly apparent at higher CuPc thicknesses, implying that the templating effect is long range.

A structure in which the 1D stacks remain intact (as suggested by electronic absorption spectroscopy), but the parallel plane separation is reduced to around $3.3 \AA$ (as suggested by XRD) is proposed in Fig. 3. Here the initial layer of CuPc molecules are forced to lie flat by the strong $\pi-\pi$ interactions with the flat lying PTCDA interlayer, with subsequent layers also forced into the same orientation. The lateral displacement of the subsequent layers remains equal to that in the 1D stacks in the $\alpha$-phase herringbone structure, resulting in a tilting of the stacking axis by around $26.5^{\circ}$ from the surface normal. In this orientation the diffraction plane parallel to the surface has an interplanar separation equal to the perpendicular separation between molecules in a stack, in this case $\sim 3.4 \AA$, which correlates well to the position of the observed XRD peak. This structure is consistent with that seen for PTCDA $/ \mathrm{H}_{2} \mathrm{Pc}^{14}$ A series of multilayer OPV devices were grown of structure ITO $/ x \AA$ $\mathrm{PTCDA} / 300 \AA \mathrm{CuPc} / 400 \AA \mathrm{C}_{60} / 100 \AA \mathrm{BCP} / \mathrm{Al}$, with $0 \AA$ $\leqslant x \leqslant 50 \AA$. CuPc and $\mathrm{C}_{60}$ layer thicknesses were selected as those optimized previously. ${ }^{6}$ Figure 4 (a) shows the $J-V$ curves for this series of OPV cells, while the inset at Fig. 4(a) displays an energy level diagram for these devices. Fig- (a)

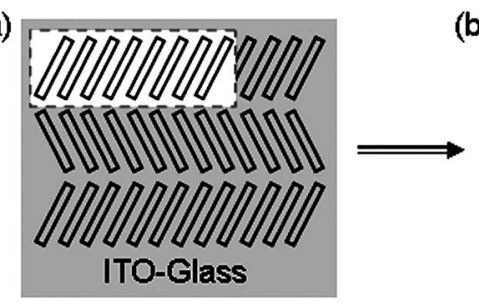

(b)

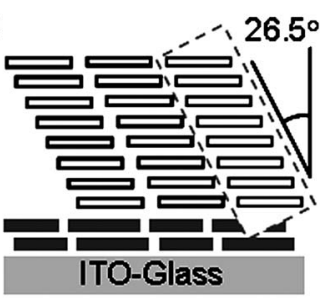

FIG. 3. A schematic showing the structure of (a) an $\alpha-\mathrm{CuPc}$ film and (b) a templated $\mathrm{CuPc}$ film with a PTCDA underlayer. The intrastack geometry, highlighted by the dashed rectangle, remains the same, but the stacking axis is rotated through approximately $90^{\circ}$. 

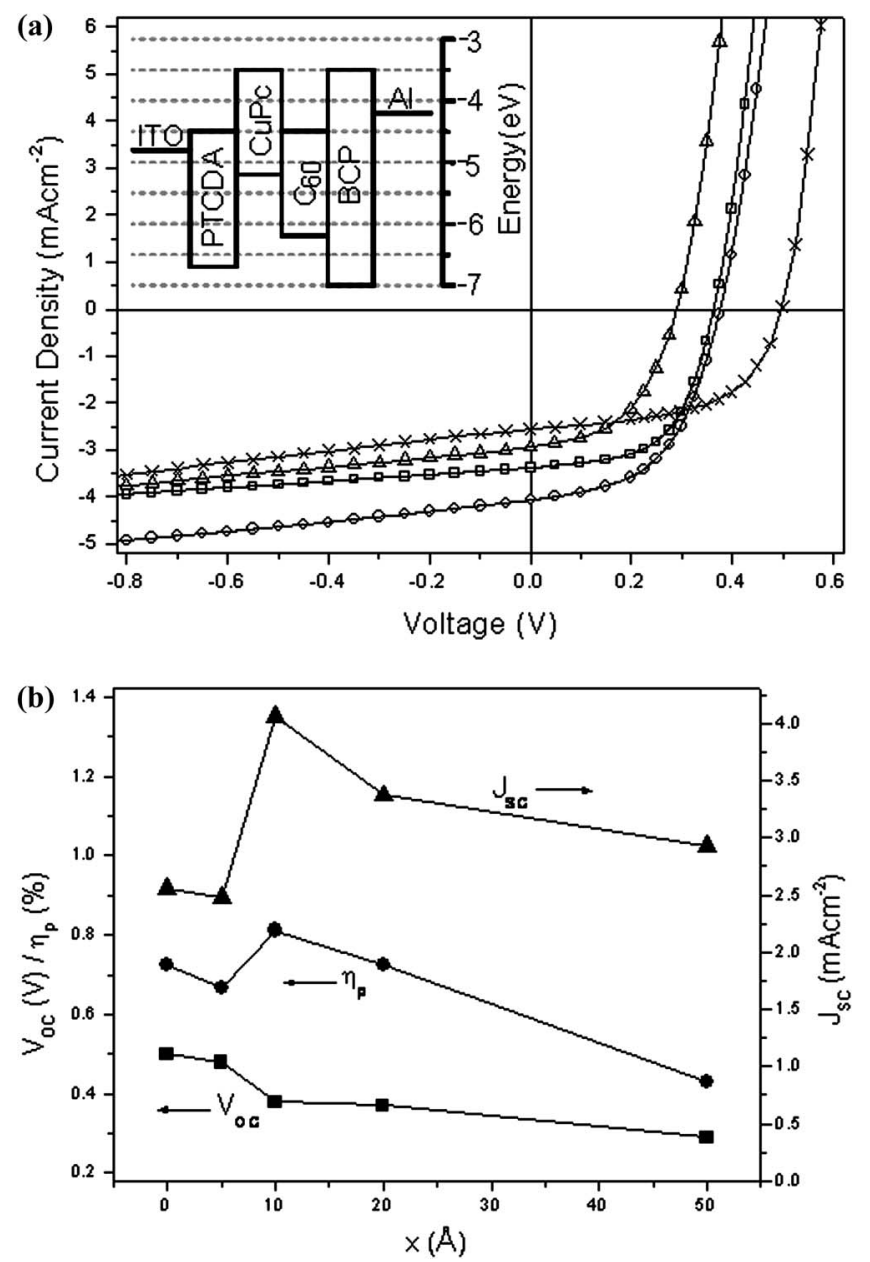

FIG. 4. (a) J-V curves for a series of ITO/ $x \AA \mathrm{PTCDA} / 300 \AA \mathrm{CuPc} / 400 \AA$ $\mathrm{C}_{60} / 100 \AA \mathrm{BCP} / \mathrm{Al}$ devices, $x=0 \AA$ (crosses), $10 \AA$ (circles), $20 \AA$ (squares), and $50 \AA$ (triangles), (a, inset) an energy level diagram for the devices, and (b) device parameters for the series; $V_{\mathrm{OC}}$ (squares), $J_{\mathrm{SC}}$ (triangles) and $\eta_{p}$ (circles).

ure 4(b) shows the trend of short circuit current density $\left(J_{\mathrm{SC}}\right)$, open circuit voltage $\left(V_{\mathrm{OC}}\right)$, and power conversion efficiency $\left(\eta_{p}\right)$ obtained under AM1.5 solar illumination as a function of PTCDA interlayer thickness.

In the absence of the PTCDA interlayer, the standard (nonoptimized) heterojunction $\mathrm{CuPc} / \mathrm{C}_{60}$ device affords a short circuit current density of $J_{\mathrm{SC}}=2.56 \mathrm{~mA} \mathrm{~cm}^{-2}$ and an open-circuit voltage of $V_{\mathrm{OC}}=0.5 \mathrm{~V}$, leading to an overall power conversion efficiency of $\eta_{p}=0.73 \%$. Inclusion of a $50 \AA$ thick PTCDA layer prior to the $\mathrm{CuPc} / \mathrm{C}_{60}$ heterostructure gives rise to a significant increase in $J_{\mathrm{SC}}$ to $2.93 \mathrm{~mA} \mathrm{~cm}^{-2}$, which we believe is due to the improved transport in the templated CuPc layer as a consequence of the modified stacking direction. Unfortunately, the improved current density is accompanied by a $40 \%$ fall in $V_{\mathrm{OC}}$, consistent with appreciable charge buildup at the PTCDA/CuPc interface, resulting in an overall drop in the power conversion efficiency to $\eta_{p}=0.43 \%$. The offset in highest occupied molecular orbital levels results in a barrier for hole extraction of $\sim 1.5 \mathrm{eV}$, two orders of magnitude greater than thermal energy at room temperature. Hole transport across the PTCDA layer would, therefore, only be expected through pinholes and defects in the structure or by tunneling through areas of low film thickness due to the roughness of the layer.

Reduction of the PTCDA layer thickness $x$ results in a gradual increase in $J_{\mathrm{SC}}$ and $V_{\mathrm{OC}}$ and, hence, $\eta_{p}$, with the optimum PTCDA thickness found to be $10 \AA$. At this thickness, $V_{\mathrm{OC}}$ approaches $0.4 \mathrm{~V}$, still considerably lower than the standard device suggesting that, as expected, charge buildup is still significant even at such low thicknesses due to the difficulty of charges crossing the PTCDA layer. However, $J_{\mathrm{SC}}$ reaches $4.06 \mathrm{~mA} \mathrm{~cm}^{-2}$, an increase of almost $60 \%$ over the standard device and sufficient to afford an improvement to $\eta_{p}=0.81 \%$. Further reduction of the PTCDA layer thickness to $5 \AA$ leads to slightly lower $J_{\mathrm{SC}}$ and $V_{\mathrm{OC}}$ values than the non-templated device suggesting that $x \approx 10 \AA$ is the critical PTCDA thickness to provide the necessary templating effect to the $\mathrm{CuPc}$ layer.

The results presented here show how, in principle, charge collection can be improved by aligning the stacking axis of molecular columns with the desired direction of charge transport. While PTCDA is clearly not the ideal surface modification in terms of energy level alignment for hole injection, the effect of templating on charge transport through the CuPc layer is clear to see. A $60 \%$ improvement in $J_{\mathrm{SC}}$, despite sufficient charge buildup to cause a $20 \%$ reduction in $V_{\mathrm{OC}}$, suggests the potential for a significant increase in OPV performance with a more suitable surface modification layer. Future work using templating layers with more appropriate energy levels will lead to significant improvements in device performance.

${ }^{1}$ C. W. Tang, Appl. Phys. Lett. 48, 183 (1986).

${ }^{2}$ S. R. Forrest, L. Y. Leu, F. F. So, and W. Y. Yoon, J. Appl. Phys. 66, 5908 (1989).

${ }^{3}$ P. Peumans, V. Bulovic, and S. R. Forrest, Appl. Phys. Lett. 76, 2650 (2000).

${ }^{4}$ P. Sullivan, S. Heutz, S. M. Schultes, and T. S. Jones, Appl. Phys. Lett. 84, 1210 (2004).

${ }^{5}$ J. G. Xue, B. P. Rand, S. Uchida, and S. R. Forrest, Adv. Mater. (Weinheim, Ger.) 17, 66 (2005).

${ }^{6}$ S. Heutz, P. Sullivan, B. M. Sanderson, S. M. Schultes, and T. S. Jones, Sol. Energy Mater. Sol. Cells 83, 229 (2004).

${ }^{7}$ S. Nespurek, H. Podlesak, and C. Hamann, Thin Solid Films 249, 230 (1994).

${ }^{8}$ S. Heutz, S. M. Bayliss, R. L. Middleton, G. Rumbles, and T. S. Jones, J. Phys. Chem. B 104, 7124 (2000).

${ }^{9}$ K. Xiao, Y. Q. Liu, G. Yu, and D. B. Zhu, Synth. Met. 137, 991 (2003).

${ }^{10}$ R. D. Gould, Thin Solid Films 125, 63 (1985).

${ }^{11}$ A. J. Lovinger, S. R. Forrest, M. L. Kaplan, P. H. Schmidt, and T. Venkatesan, J. Appl. Phys. 55, 476 (1984).

${ }^{12}$ S. Heutz, A. J. Ferguson, G. Rumbles, and T. S. Jones, Org. Electron. 3, 119 (2002)

${ }^{13}$ S. Heutz, R. Cloots, and T. S. Jones, Appl. Phys. Lett. 77, 3938 (2000).

${ }^{14}$ S. Heutz and T. S. Jones, J. Appl. Phys. 92, 3039 (2002).

${ }^{15}$ S. Yim, S. Heutz, and T. S. Jones, Phys. Rev. B 67, 165308 (2003).

${ }^{16}$ A. A. Ebert and H. B. Gottlieb, J. Am. Chem. Soc. 74, 2806 (1952).

${ }^{17}$ Rudiono, F. Kaneko, and M. Takeuchi, Appl. Surf. Sci. 142, 598 (1999).

${ }^{18}$ M. M. El-Nahass, F. S. Bahabri, A. A. AL Ghamdi, and S. R. Al-Harbi, Egypt. J. Solids 25, 307 (2002).

${ }^{19}$ B. P. Rand, J. Xue, S. Uchida, and S. R. Forrest, J. Appl. Phys. 98, 124902 (2005). 